Research Paper

\title{
Outcomes of cancer therapy administered to treatment-naïve lung cancer patients in the intensive care unit
}

\author{
Yen-Fu Chen'1, Jou-Wei Lin'1, Chao-Chi Ho², Ching-Yao Yang22, Chia-Hao Chang3, Tao-Min Huang1, \\ Chung-Yu Chen ${ }^{1 凶}$, Kuan-Yu Chen², Jin-Yuan Shih², Chong-Jen Yu ${ }^{2}$ \\ 1. Department of Internal Medicine, National Taiwan University Hospital Yun-Lin Branch, No.579, Sec. 2, Yunlin Rd., Douliou City, Yunlin County 640, \\ Taiwan (R.O.C.) \\ 2. Department of Internal Medicine, National Taiwan University Hospital, No.7, Zhongshan South Road, Taipei 100, Taiwan (ROC) \\ 3. Department of Internal Medicine, National Taiwan University Hospital Hsin-Chu Branch, No. 25, Lane 442, Sec. 1, Jingguo Rd., Hsinchu City 30059, Taiwan \\ (R.O.C.) \\ $\triangle$ Corresponding author: Chung-Yu Chen, MD, E-mail: c8101147@ms16.hinet.net; Phone: +886-5-5323911-5675; Fax: +886-5-5351377 \\ (c) Ivyspring International Publisher. This is an open access article distributed under the terms of the Creative Commons Attribution (CC BY-NC) license \\ (https://creativecommons.org/licenses/by-nc/4.0/). See http://ivyspring.com/terms for full terms and conditions.
}

Received: 2016.11.01; Accepted: 2017.05.03; Published: 2017.07.05

\begin{abstract}
Objectives: Therapy outcomes for newly diagnosed, critically ill lung cancer patients have seldom been evaluated. This study evaluated therapy outcomes for treatment-naive lung cancer patients in the intensive care unit (ICU).

Materials and Methods: Patients were excluded if they had previously received lung cancer treatment, such as systemic chemotherapy, targeted therapy, radiotherapy, or surgical lung resection before ICU admission. The therapeutic strategies for the treatment-naive patients were determined while they were in the ICU. The patients' demographic data, clinical outcomes, and treatment-related toxicities were analyzed.

Results: Newly diagnosed lung cancer patients $(n=72)$ who did not receive any anticancer treatment before ICU admission were included. Most patients had locally advanced disease, and 61 $(84.7 \%)$ required intensive care due to cancer-related events. In the ICU, 24 (33.3\%) patients received chemotherapy, 24 (33.3\%) received epidermal growth factor receptor-tyrosine kinase inhibitor (EGFR-TKI) therapy and 24 (33.3\%) received best supportive care (BSC). Patients receiving chemotherapy or EGFR-TKIs in the ICU demonstrated better ICU $(p=0.011)$ and in-hospital $(p=0.034)$ survival than those receiving BSC only. Among patients requiring mechanical ventilation, those receiving chemotherapy had higher weaning rates than those receiving EGFR-TKIs or BSC $(p=0.002)$. In multivariate analysis, receipt of chemotherapy (hazard ratio $[H R], 0.443 ; p=0.083)$ and mechanical ventilation $(H R, 0.270 ; p=0.022)$ were significantly associated with longer ICU survival after adjusting for clinical factors.
\end{abstract}

Conclusions: Anticancer therapy in the ICU might provide better short-term ICU survival for treatment-naïve, critically ill lung cancer patients.

Key words: Treatment-naïve lung cancer; Intensive care unit; Non-small cell lung cancer; Epidermal growth factor receptor; Tyrosine kinase inhibitor; Small cell lung cancer; Best supportive care

\section{Introduction}

Lung cancer remains a leading cause of cancer-related mortality among men and women, worldwide [1]. Patients may present in a critically ill state due to malignancy-related complications or due to underlying co-morbidities. Such patients, admitted to the intensive care unit (ICU), have mortality rates of $42-73 \%$ [2-6]. Although critical care advances have improved the survival of these critically ill patients, 
not all lung cancer patients have derived benefit from intensive care [7]. Several retrospective studies identified factors associated with the poor ICU outcomes for these patients, including requiring mechanical ventilation $[3,4,6,8-10]$, poor pre-event performance status (PS) $[2,6,11]$, high admission Acute Physiologic and Chronic Health Evaluation (APACHE) III scores with vasopressor use [8], and refractory disease $[4,6,8,12]$. Nevertheless, treatment outcomes and mortality predictors remain unclear for treatment-naïve lung cancer patients requiring intensive care.

Treatment of locally advanced and metastatic lung cancers, including targeted therapy, chemotherapy, and best supportive care (BSC), are usually administered according to age, patient PS, cancer cell type, and molecular status [13,14]. Patients with extremely poor PS, including critically ill patients, are usually excluded from anticancer therapy prospective clinical trials and their first-line treatment options are usually limited to BSC [13]. Thus, the role of cancer therapies for treatment-naïve, critically lung cancer patients remain unclear.

Only a few reports have focused on the management of treatment-naïve lung cancer patients admitted to the ICU due to cancer-related complications or other critical conditions [15, 16]. Therefore, treatment strategies for newly diagnosed, treatment-naive lung cancer patients in the ICU have never been compared. The present study retrospectively assessed the clinical factors and management (including anticancer therapies and BSC) outcomes for treatment-naïve, lung cancer patients under intensive care. We also investigated the impact of different treatment strategies, and their related side effects, on clinical outcomes.

\section{Material and Methods}

\section{Study design and patients}

We retrospectively reviewed the electronic medical records of newly diagnosed lung cancer inpatients in the ICUs of National Taiwan University Hospital (NTUH), Taipei, and the NTUH Yunlin-branch, between January 1, 2001 and September 1, 2013. Patients were excluded if they had received any lung cancer treatment, including systemic chemotherapy, targeted therapy, radiotherapy, or surgical lung resection, before ICU admission. Patients admitted to medical or surgical ICUs due to complications arising from previous anticancer therapies (such as chemotherapy and targeted therapy) or postoperative care were also excluded. For patients meeting the inclusion criteria, but with multiple ICU admissions, only the first admission was included in the analysis. The management and treatment strategies (including BSC, chemotherapy, and target therapy) for treatment-naïve lung cancer patients in the ICU were made by intensivists, often after discussion with oncologists. Patients who had never smoked or who had smoked $<100$ cigarettes in their lifetime were categorized as non-smokers [17]. The study protocol was approved by the hospitals' Research Ethics Committees and the need for informed consent was waived.

\section{Data collection and outcomes}

After enrollment, demographics and baseline characteristics such as age, sex, co-morbidity, pre-ICU PS (bedridden or non-bedridden), ICU admission diagnosis, and illness severity upon ICU admission (APACHE II score [18]) were recorded for all patients. Other clinical data, including cancer stage [19], lung cancer histologic type (non-small cell lung cancer [NSCLC] or small cell lung cancer [SCLC]) [20], molecular status, and metastases sites were recorded. Extensive cancer disease was defined as stage IIIB or IV for NSCLC and as extensive-stage for SCLC [19]. We also classified the primary reasons for ICU admission into non-lung cancer-related (e.g., severe sepsis/septic shock, pneumonia, chronic obstructive pulmonary disease with acute exacerbation, cardiac arrhythmia and acute myocardial infarction) and lung cancer-induced (e.g., tumor-related critical airway and obstructive pneumonitis, superior vena cava syndrome, and pulmonary embolism) events.

The lung cancer therapy regimens in the ICU and treatment-related toxicities were recorded [21]. Patients were classified according to their management into the chemotherapy, epidermal growth factor receptor (EGFR)-tyrosine kinase inhibitors (TKIs), or BSC (no specific cancer therapy) group. Data regarding ICU management of the patients, such as the use of mechanical ventilation, hemodialysis, vasopressors use, and do-not resuscitation (DNR) orders, were also collected. Additionally, the main study outcomes and the lengths of ICU and hospital stays were also assessed. Survival times were calculated as the interval between the date of ICU/hospital admission and the death date, last follow-up date, or the final follow-up prior to September 2013, whichever came first. We also analyzed the impact of clinical factors associated with study outcomes.

\section{Statistical analysis}

All categorical variables were analyzed using Pearson's $\chi^{2}$ tests, except where a small sample size $(<$ 5) required the use of Fisher's exact test. One-way 
analysis of variance was used to analyze differences in patient characteristics among the three treatment strategy groups. The ICU and hospital survival times were plotted using the Kaplan-Meier method and compared using the log-rank test. Multivariate analyses for ICU and hospital survival were performed using the Cox proportional hazards model. The hazard ratios (HRs), 95\% confidence intervals (CIs), and p-values are reported. Statistical significance was set at a two-sided $p<0.05$. All analyses were performed using SPSS version 18.0 (SPSS, Chicago, IL).

\section{Results}

\section{Patient characteristics}

The electronic medical records of all 1181 lung cancer patients ever admitted to the ICUs were initially screened. Of these admissions, 421 patients admitted to the ICU for postoperative and post-procedure care were excluded. Another 356 patients were excluded due to prior chemotherapy receipt. In addition, 232 patients who had received molecularly targeted agents, 21 patients who had received radiotherapy, 60 patients with prior lung resections due to lung cancer, and 19 patients with incomplete data were excluded. Finally, 72 newly diagnosed lung cancer patients without prior cancer therapy were admitted to the ICU and were included in the analyses.

As shown in Table 1, the mean patient age was 68.8 years, and $56(77.8 \%)$ were male. NSCLC was diagnosed in $58(80.6 \%)$ patients (stage IIIA [n $=1]$, stage IIIB [ $n=6]$, or stage IV [ $n=51]$ ) and SCLC was diagnosed in 14 (19.4\%) patients (all extensive stage). Among the advanced lung cancer patients, 49 (68.1\%) had at least two metastatic sites. A positive EGFR mutation was identified in only two adenocarcinoma patients (an exon 19 deletion and an L858R mutation); the remainder were either wild type $(n=15)$ or had an unknown $(\mathrm{n}=55)$ mutation status. The mean APACHE II scores were 20.6 in the chemotherapy group and 21.2 in the EGFR-TKI group, not significantly higher than for the BSC group (17.9; $\mathrm{p}=$ 0.295). A total of $47(65.3 \%)$ patients were smokers, with a significantly higher proportion of patients in the chemotherapy group being smokers $(p=0.016)$.

Table 1. Baseline characteristics of treatment-naïve lung cancer patients treated in the intensive care unit

\begin{tabular}{|c|c|c|c|c|c|}
\hline & All patients & Best supportive care & Chemotherapy & EGFR-TKI & p-value \\
\hline Number & 72 & 24 & 24 & 24 & \\
\hline Age, years (mean \pm SD) & $68.8 \pm 12.8$ & $70.7 \pm 13.4$ & $70.5 \pm 10.0$ & $65.1 \pm 14.4$ & 0.238 \\
\hline Age, > 65 years $(\mathrm{n}[\%])$ & $48(66.7)$ & $17(70.8)$ & $18(75.0)$ & $13(54.2)$ & 0.269 \\
\hline Sex, male (n $[\%])$ & $56(77.8)$ & $19(79.2)$ & $22(91.7)$ & $15(62.5)$ & 0.051 \\
\hline APACHE II score (mean \pm SD) & $19.9 \pm 7.8$ & $17.9 \pm 8.8$ & $20.6 \pm 5.9$ & $21.2 \pm 8.4$ & 0.295 \\
\hline Smoking (n [\%]) & $47(65.3)$ & $12(50.0)$ & $21(87.5)$ & $14(58.3)$ & 0.016 \\
\hline \multicolumn{6}{|l|}{ Pre ICU performance (n [\%]) } \\
\hline Non-bedridden & 61 & $18(75)$ & $22(91.7)$ & $21(87.5)$ & 0.248 \\
\hline bedridden & 11 & $6(25)$ & $2(8.3)$ & $3(12.5)$ & \\
\hline \multicolumn{6}{|l|}{ Histologic type } \\
\hline $\operatorname{SCLC~(n~[\% ])~}$ & $14(19.4)$ & $3(12.5)$ & $11(45.8)$ & $0(0)$ & $<0.001$ \\
\hline NSCLC (n [\%]) & $58(80.6)$ & $21(87.5)$ & $13(54.2)$ & $24(100)$ & \\
\hline Adenocarcinoma & 45 & 17 & 6 & 22 & \\
\hline Squamous cell carcinoma & 8 & 3 & 4 & 1 & \\
\hline NSCLC-NOS & 2 & 0 & 2 & 0 & \\
\hline Sarcomatoid carcinoma & 2 & 0 & 1 & 1 & \\
\hline Pleomorphic carcinoma & 1 & 1 & 0 & 0 & \\
\hline \multicolumn{6}{|l|}{ Clinical stage } \\
\hline I-IIIA or limited stage (n [\%]) & $1(1.4)$ & $1(4.2)$ & $0(0)$ & $0(0)$ & 0.363 \\
\hline IIIB-IV/extensive stage (n [\%]) & $71(98.6)$ & $23(95.8)$ & $24(100)$ & $24(100)$ & \\
\hline \multicolumn{6}{|l|}{ EGFR mutation (n [\%]) } \\
\hline Wild type or not-available & $70(97.2)$ & $23(95.8)$ & $24(100)$ & $23(95.8)$ & 0.598 \\
\hline Mutation & $2(2.8)$ & $1(4.2)$ & $0(0)$ & $1(4.2)$ & \\
\hline \multicolumn{6}{|l|}{ Metastasis sites } \\
\hline $0-1(\mathrm{n}[\%])$ & $23(31.9)$ & $6(25)$ & $13(54.2)$ & $4(16.7)$ & 0.014 \\
\hline$>2(\mathrm{n}[\%])$ & $49(68.1)$ & $18(75)$ & $11(45.8)$ & $20(83.3)$ & \\
\hline \multicolumn{6}{|l|}{ Co-morbidities } \\
\hline Diabetes mellitus (n [\%]) & $20(27.8)$ & $7(29.2)$ & $8(33.3)$ & $5(20.8)$ & 0.616 \\
\hline Hypertension (n [\%]) & $30(41.7)$ & $10(41.7)$ & $10(41.7)$ & $10(41.7)$ & 1.000 \\
\hline COPD (n [\%]) & $20(27.8)$ & $8(33.3)$ & $7(29.2)$ & $5(20.8)$ & 0.616 \\
\hline Chronic kidney disease (n [\%]) & $19(26.4)$ & $5(20.8)$ & $7(29.2)$ & $7(29.2)$ & 0.751 \\
\hline Cardiovascular disease (n [\%]) & $12(16.7)$ & $5(20.8)$ & $4(16.7)$ & $3(12.5)$ & 0.741 \\
\hline
\end{tabular}


Table 2. Characteristics and outcomes of treatment-naïve lung cancer patient treated in an intensive care unit

\begin{tabular}{|c|c|c|c|c|c|}
\hline & All patients & $\begin{array}{l}\text { Best supportive } \\
\text { care }\end{array}$ & Chemotherapy & EGFR-TKI & p-value \\
\hline Number & 72 & 24 & 24 & 24 & \\
\hline \multicolumn{6}{|l|}{ Primary reasons for ICU admission } \\
\hline Non-lung cancer related events (n [\%]) & $11(15.3)$ & $5(20.8)$ & $1(4.2)$ & $5(20.8)$ & 0.180 \\
\hline Pneumonia with respiratory failure (n) & 3 & 0 & 0 & 3 & \\
\hline Septic shock $(n)$ & 3 & 0 & 1 & 2 & \\
\hline COPD with acute exacerbation (n) & 2 & 2 & 0 & 0 & \\
\hline Acute coronary syndrome (n) & 1 & 1 & 0 & 0 & \\
\hline Congestive heart failure (n) & 1 & 1 & 0 & 0 & \\
\hline Cardiac arrest $(\mathrm{n})$ & 1 & 1 & 0 & 0 & \\
\hline Lung cancer-related events (n [\%]) & $61(84.7)$ & $19(79.2)$ & $23(95.8)$ & $19(79.2)$ & \\
\hline Obstructive pneumonitis (n) & 27 & 9 & 13 & 5 & \\
\hline Cardiac tamponade (n) & 9 & 6 & 0 & 3 & \\
\hline Massive malignant pleural effusion (n) & 6 & 2 & 2 & 2 & \\
\hline Critical airway $(n)$ & 7 & 1 & 4 & 2 & \\
\hline Lymphangitic carcinomatosis (n) & 7 & 0 & 2 & 5 & \\
\hline Superior vena cava syndrome (n) & 2 & 1 & 1 & 0 & \\
\hline Pulmonary embolism (n) & 3 & 0 & 1 & 2 & \\
\hline Mechanical ventilator use (n [\%]) & $47(65.3)$ & $11(45.8)$ & $19(79.2)$ & $17(70.8)$ & 0.041 \\
\hline Weaning off MV (n [\%]) & $12 / 47(25.5)$ & $1 / 11(9.1)$ & $10 / 19(52.6)$ & $1 / 17(5.9)$ & 0.002 \\
\hline Acute kidney injury (n [\%]) & $27(37.5)$ & $7(29.2)$ & $11(48.8)$ & $9(37.5)$ & 0.491 \\
\hline Sepsis in ICU (n [\%]) & $54(75.0)$ & $13(54.2)$ & $20(83.3)$ & $20(83.3)$ & 0.030 \\
\hline Anticancer treatment related & $20(27.8)$ & - & $11(45.8)$ & $9(37.5)$ & 0.385 \\
\hline Vasopressor use (n [\%]) & $31(43.1)$ & $11(45.8)$ & $8(33.3)$ & $12(50.0)$ & 0.479 \\
\hline DNR order in ICU (n [\%]) & $43(59.7)$ & $17(70.8)$ & $10(41.7)$ & $16(66.7)$ & 0.084 \\
\hline ICU mortality (n [\%]) & $31(43.1)$ & $12(50)$ & $6(25)$ & $13(54.2)$ & 0.088 \\
\hline In-hospital mortality (n [\%]) & $46(63.9)$ & $15(62.5)$ & $14(58.3)$ & $17(70.8)$ & 0.656 \\
\hline
\end{tabular}

\section{Main reasons for ICU admission}

The reasons for ICU admission are listed in Table 2. Cancer-induced events were the main reasons for ICU admission (61/72, 84.7\%), including obstructive pneumonitis $(\mathrm{n}=27)$, cardiac tamponade $(\mathrm{n}=9)$, massive pleural effusion ( $n=7)$, critical airway $(n=7)$, lymphangitic carcinomatosis $(n=7)$, superior vena cava syndrome $(\mathrm{n}=2)$, and pulmonary embolism $(\mathrm{n}=$ 3). The other 11 patients were admitted to the ICU due to non-cancer related events. Diagnoses of pneumonia with respiratory failure and septic shock were the most common reasons for ICU admission.

\section{ICU patient management}

After ICU admission, 48 patients (66.7\%) received systemic anticancer therapy, with 24 patients receiving EGFR-TKI therapy (gefitinib or erlotinib) and 24 patients receiving other first-line chemotherapies (Table 3). One squamous cell carcinoma patient and two SCLC patients in the chemotherapy group received concurrent chemotherapy and radiotherapy.

Forty-seven $(65.3 \%)$ patients demonstrated acute respiratory failure and received mechanical ventilation upon admission, including $11(45.8 \%)$ in the BSC group, $19(79.2 \%)$ in the chemotherapy group, and $17(70.8 \%)$ in the EGFR-TKI group $(p=0.041)$. Ten (8 SCLC patients and 2 NSCLC patients) of the 19
(52.6\%) patients in the chemotherapy group were successfully weaned off mechanical ventilation in the ICU. The chemotherapy group had a higher weaning rate than did the EGFR-TKI $(5.9 \%)$ or BSC $(9.1 \%)$ group $(p=0.002)$.

More sepsis events were noted in the treatment groups (20[83.3\%]) than in the BSC group (13[54.2\%]) with a $\mathrm{p}$ value 0.030 . Nevertheless, the anticancer treatment related sepsis events were similar in chemotherapy group (11[45.8\%] and EGFR-TKI group $(9[37.5 \%])(p=0.385)$ (Table 2$)$. Thirty-one $(43.1 \%)$ of the ICU patients received vasopressors in the ICU, with similar proportions of cases in the treatment and BSC groups $(p=0.479)$. DNR orders were provided by two-thirds of the patients during their ICU course, and were more commonly observed in the BSC and EGFR-TKI groups $(\mathrm{p}=0.084)$.

\section{Clinical outcomes}

During their ICU course, $6(25 \%)$ patients in the chemotherapy group, $13(54.2 \%)$ in the EGFR-TKI group, and $12(50 \%)$ in the BSC group $(p=0.088)$ died. The median ICU survival times were 48, 28, and 11 days for patients in the chemotherapy, EGFR-TKI, and BSC groups, respectively $(\mathrm{p}=0.011)$ (Figure 1). The in-hospital mortality rate was $58.3 \%(14 / 24)$ for the chemotherapy group, $70.8 \%(17 / 24)$ for the targeted therapy group, and $62.5 \%(15 / 24)$ for the BSC group $(p=0.656)$. The median in-hospital 


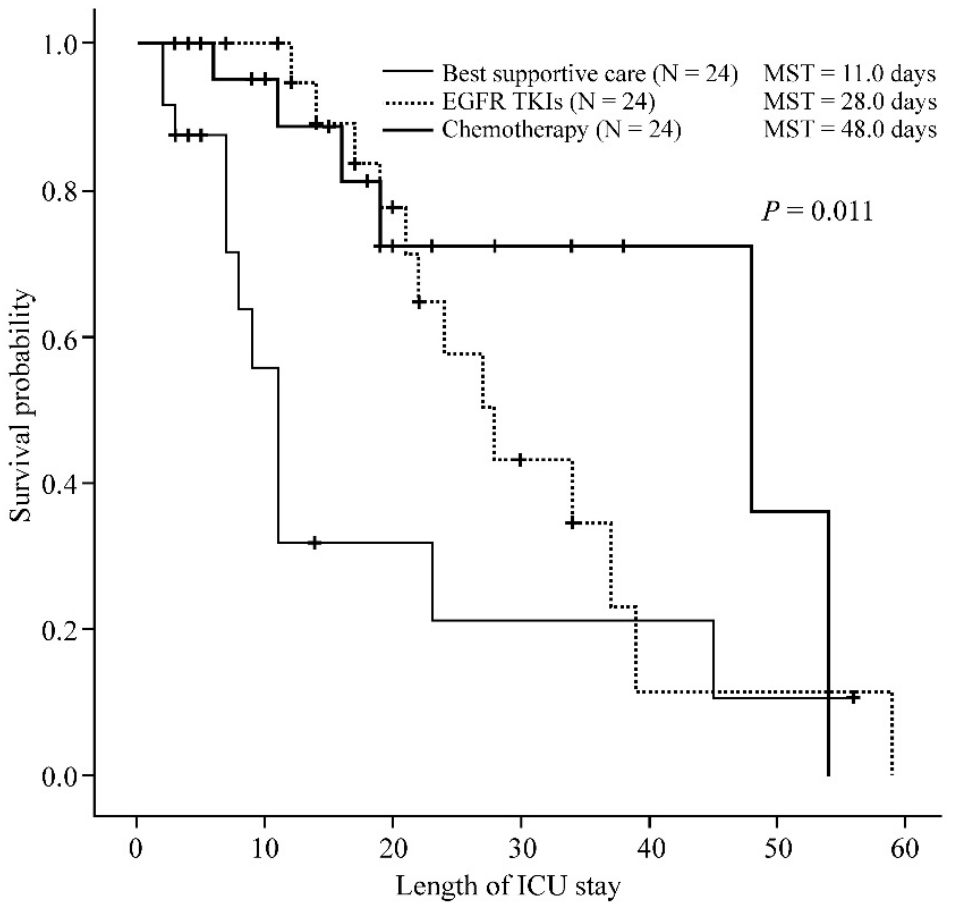

Figure 1. Outcomes of treatment-naïve lung cancer patients receiving different treatment strategies in the intensive care unit. Kaplan-Meier curves of intensive care unit (ICU) survival of treatment-naïve lung cancer patients $(n=72)$ admitted to the ICU, receiving different treatment strategies. The patients treated with chemotherapy (thick line), or epidermal growth factor receptor (EGFR) and tyrosine kinase inhibitor (TKI) (thick dotted line) in the ICU demonstrate longer ICU survival than patients receiving best supportive care (thin line) $(p=0.011)$. There was no significant difference in ICU survival ( 28 days vs. 48 days, $p=0.374$ ) between the EGFR-TKI and chemotherapy groups. The $p$-value was calculated using the log-rank test. MST, median survival time.

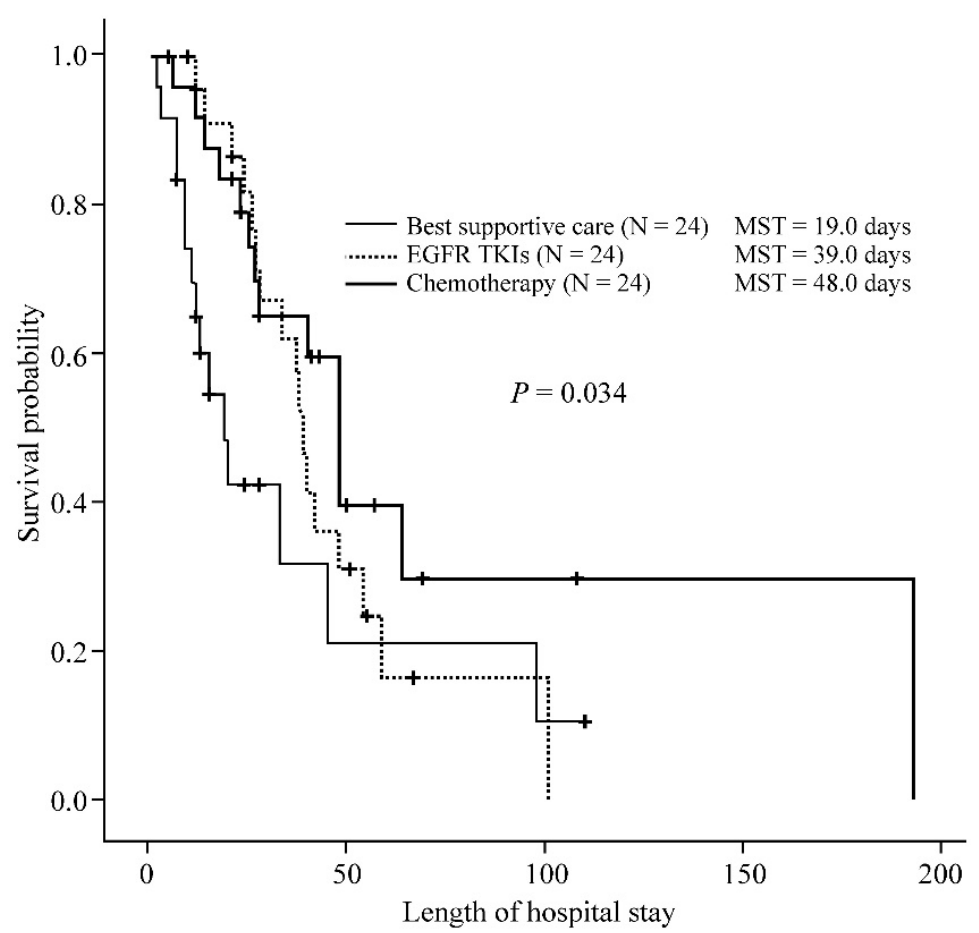

Figure 2. Hospital survival of treatment-naïve lung cancer patients receiving different treatment strategies in the intensive care unit. Kaplan-Meier curves of in-hospital survival of treatment-naïve lung cancer patients $(n=72)$ admitted to the intensive care unit (ICU), undergoing different treatment strategies. Patients receiving chemotherapy (thick line), or epidermal growth factor receptor (EGFR) and tyrosine kinase inhibitor (TKI) (thick dotted line) therapy in the ICU had significantly longer in-hospital survival than patients receiving best supportive care (thin line) $(p=0.034)$. However, there was no significant difference in the in-hospital survival ( 28 days vs. 48 days, $p=0.374$ ) between the EGFR-TKI and chemotherapy groups. The $\mathrm{p}$-value was calculated using the log-rank test. MST, median survival time. survival times were 48,39 , and 19 days for patients in the chemotherapy, EGFR-TKI, and BSC groups, respectively $(\mathrm{p}=0.034)$ (Figure 2). There were no significant differences in ICU survival (28 days vs. 48 days, $\mathrm{p}=0.374$ ) or in-hospital survival (39 days vs. 48 days, $p$ $=0.269$ ) between the EGFR-TKI and chemotherapy groups.

After adjusting for clinical factors and patient management (sex, smoking status, histologic type, metastasis sites, mechanical ventilator use, systemic anticancer therapy, and DNR orders), the multi-variant analysis for ICU survival showed that patients receiving chemotherapy $(\mathrm{HR}, 0.202 ; \mathrm{p}=0.012)$ and mechanical ventilation $(\mathrm{HR}, 0.270 ; \mathrm{p}=$ 0.022 ) were significantly associated with longer ICU survivals (Table 3). The multi-variant analysis for hospital survival showed that patients receiving chemotherapy (HR, 0.443; $\mathrm{p}=0.083$ ) appeared to have relatively longer hospital survival than BSC group. However, DNR orders (HR, 4.516; $\mathrm{p}=$ 0.001 ) were significantly associated with shorter hospital survival times.

Table 4 shows the treatment side effects and regimens. Patients who received chemotherapy, especially platinum-based regimens, had higher risks of grade 3 or 4 neutropenia $(10 / 24,41.6 \%)$ and thrombocytopenia $(5 / 24,20.8 \%)$ than did patients in the EGFR-TKI group. Nevertheless, three patients $(3 / 24,12.5 \%)$ in the EGFR-TKI group had suspicious gefitinib-related interstitial pneumonitis.

\section{Discussion}

This is the first study to specifically focus on the outcomes of different anticancer strategies of treatment-naïve lung cancer (including SCLC and NSCLC) patients admitted to the ICU. Therapy involving either chemotherapy or EGFR-TKIs might provide better ICU and in-hospital survival times than BSC for these patients. Moreover, among patients requiring mechanical ventilation, those who received chemotherapy in the ICU seemed to have higher weaning rates than did those receiving EGFR-TKIs and BSC. However, patients receiving chemotherapy and targeted therapy in the ICU also have higher risks of treatment-related side effects, but not mortality, than those receiving BSC. 
Table 3. Multivariate analysis of clinical factors associated with intensive care unit survival of treatment-naïve lung cancer patients

\begin{tabular}{|c|c|c|c|c|c|c|c|c|c|}
\hline \multirow[t]{2}{*}{ Clinical factors } & \multirow[t]{2}{*}{$\begin{array}{l}\text { No. of } \\
\text { patients }\end{array}$} & \multirow{2}{*}{$\begin{array}{l}\text { Median } \\
\text { ICU } \\
\text { survival } \\
\text { (days) }\end{array}$} & \multicolumn{3}{|c|}{$\begin{array}{l}\text { Univariate Multivariate analysis } \\
\text { analysis }\end{array}$} & \multirow{2}{*}{$\begin{array}{l}\text { Median } \\
\text { Hospital } \\
\text { survival } \\
\text { (days) }\end{array}$} & \multirow{2}{*}{$\begin{array}{l}\text { Univariate } \\
\text { analysis }\end{array}$} & \multicolumn{2}{|l|}{ Multivariate analysis } \\
\hline & & & p-value & HR $(95 \%$ CI) & p-value & & & HR $(95 \%$ CI) & p-value \\
\hline \multicolumn{10}{|l|}{ Anticancer therapy } \\
\hline Best supportive care & 24 & 11 & 0.011 & 1.000 & & 19 & 0.034 & 1.000 & \\
\hline EGFR-TKI & 24 & 28 & & $0.394(0.143-1.085)$ & 0.072 & 39 & & $0.610(0.263-1.412)$ & 0.248 \\
\hline Chemotherapy & 24 & 48 & & $0.202(0.058-0.699)$ & 0.012 & 48 & & $0.443(0.177-1.111)$ & 0.083 \\
\hline \multicolumn{10}{|l|}{ Gender } \\
\hline Female & 16 & 59 & 0.063 & & & 54 & 0.159 & & \\
\hline Male & 56 & 24 & & $3.310(0.771-14.209)$ & 0.107 & 37 & & $2.397(0.778-7.383)$ & 0.128 \\
\hline \multicolumn{10}{|l|}{ Smoking status } \\
\hline Never smoker & 25 & 39 & 0.286 & & & 48 & 0.478 & & \\
\hline Smoker & 47 & 23 & & 1.445 (0.463-4.507) & 0.526 & 34 & & $1.069(0.441-2.590)$ & 0.883 \\
\hline \multicolumn{10}{|l|}{ Histologic type } \\
\hline SCLC & 14 & 23 & 0.993 & & & 40 & 0.377 & & \\
\hline NSCLC & 58 & 28 & & $0.728(0.225-2.357)$ & 0.597 & 38 & & $0.804(0.317-2.039)$ & 0.645 \\
\hline \multicolumn{10}{|l|}{ Metastasis sites } \\
\hline $0-1$ & 23 & 23 & 0.586 & & & 45 & 0.372 & & \\
\hline $2-4$ & 49 & 27 & & $1.243(0.489-3.157)$ & 0.648 & 37 & & $1.581(0.753-3.321)$ & 0.226 \\
\hline \multicolumn{10}{|c|}{ Mechanical ventilator use } \\
\hline No & 25 & 12 & 0.038 & & & 40 & 0.824 & & \\
\hline Yes & 47 & 28 & & $0.270(0.088-0.827)$ & 0.022 & 38 & & $0.495(0.206-1.188)$ & 0.115 \\
\hline \multicolumn{10}{|l|}{ Sepsis event } \\
\hline No & 19 & 11 & 0.530 & & & 13 & 0.037 & & \\
\hline Yes & 53 & 27 & & $5.519(0.489-54.453)$ & 0.172 & 34 & & $3.589(0.834-15.443)$ & 0.086 \\
\hline \multicolumn{10}{|l|}{ DNR order in ICU } \\
\hline No & 29 & 54 & 0.020 & & & 101 & $<0.001$ & & \\
\hline Yes & 43 & 22 & & $2.302(0.584-9.078)$ & 0.234 & 26 & & $4.516(1.792-11.382)$ & 0.001 \\
\hline
\end{tabular}

Abbreviations: CI: confidence interval; EGFR, epidermal growth factor receptor; HR, hazard ratio; ICU, Intensive care unit; NSCLC, non-small cell lung cancer; TKI,

tyrosine-kinase inhibitor; SCLC, small cell lung cancer; DNR, do not resuscitate

Table 4. Chemotherapy and targeted therapy for 48 treatment-naïve lung cancer patients treated in the intensive care unit

\begin{tabular}{|c|c|c|c|c|c|c|c|c|c|c|c|}
\hline & \multicolumn{2}{|c|}{ Cancer cell type } & \multicolumn{7}{|c|}{ Side effects recorded in ICU } & \multirow{2}{*}{$\begin{array}{l}\text { Death } \\
\text { in ICU }\end{array}$} & \multirow{2}{*}{$\begin{array}{l}\text { Death in } \\
\text { hospital }\end{array}$} \\
\hline & $\begin{array}{l}\text { SCLC } \\
(n=11)\end{array}$ & $\begin{array}{l}\text { NSCLC } \\
(\mathrm{n}=37)\end{array}$ & $\begin{array}{l}\text { Neutropenia } \\
\text { Grade 3/4 }\end{array}$ & $\begin{array}{l}\text { Anemia } \\
\text { Grade } \\
3 / 4\end{array}$ & $\begin{array}{l}\text { Thrombocytopenia } \\
\text { Grade } 3 / 4\end{array}$ & $\begin{array}{l}\text { GI } \\
\text { toxicity } \\
\text { Grade } \\
3 / 4\end{array}$ & $\begin{array}{l}\text { Skin } \\
\text { toxicity } \\
\text { Grade } \\
3 / 4\end{array}$ & $\begin{array}{l}\text { Acute } \\
\text { kidney } \\
\text { injury }\end{array}$ & $\begin{array}{l}\text { Treatment } \\
\text {-related IP }\end{array}$ & & \\
\hline \multicolumn{12}{|l|}{ Chemotherapy $(n=24)$} \\
\hline Etoposide + cisplatin $(n=6)$ & 5 & 1 & 3 & 0 & 2 & 0 & 0 & 0 & 0 & 3 & 5 \\
\hline Etoposide + carboplatin $(n=5)$ & 4 & 1 & 4 & 1 & 2 & 2 & 0 & 0 & 0 & 0 & 1 \\
\hline Etoposide + vincristine $(\mathrm{n}=1)$ & 1 & 0 & 1 & 0 & 1 & 0 & 0 & 0 & 0 & 1 & 1 \\
\hline $\begin{array}{l}\text { Carboplatin }+ \text { pemetrexed }(n= \\
\text { 2) }\end{array}$ & 1 & 1 & 0 & 0 & 0 & 0 & 1 & 0 & 0 & 0 & 0 \\
\hline $\begin{array}{l}\text { Carboplatin }+ \text { pemetrexed }+ \\
\text { bevacizumab }(n=1)\end{array}$ & 0 & 1 & 1 & 0 & 0 & 1 & 0 & 0 & 0 & 0 & 1 \\
\hline $\begin{array}{l}\text { Gemcitabine }(\mathrm{II}) / \text { cisplatin }+ \\
\text { pemetrexed }(n=1)\end{array}$ & 0 & 1 & 0 & 0 & 0 & 0 & 0 & 0 & 0 & 0 & 1 \\
\hline Cisplatin + gemcitabine $(n=1)$ & 0 & 1 & 0 & 0 & 0 & 0 & 0 & 1 & 0 & 0 & 1 \\
\hline Cisplatin + docetaxel $(n=2)$ & 0 & 2 & 1 & 0 & 0 & 1 & 0 & 0 & 0 & 0 & 0 \\
\hline Paclitaxel/gemcitabine $(n=1)$ & 0 & 1 & 0 & 0 & 0 & 0 & 0 & 0 & 0 & 1 & 1 \\
\hline Docetaxel $(n=2)$ & 0 & 2 & 0 & 0 & 0 & 1 & 0 & 0 & 0 & 1 & 1 \\
\hline Gemcitabine $(n=1)$ & 0 & 1 & 0 & 0 & 0 & 0 & 0 & 0 & 0 & 0 & 1 \\
\hline Vinorelbine $(\mathrm{n}=1)$ & 0 & 1 & 0 & 0 & 0 & 0 & 0 & 0 & 0 & 0 & 1 \\
\hline \multicolumn{12}{|l|}{ EGFR-TKI therapy $(n=24)$} \\
\hline Gefitinib $(n=16)$ & 0 & 16 & 0 & 0 & 0 & 1 & 0 & 0 & 3 & 8 & 10 \\
\hline Erlotinib $(\mathrm{n}=6)$ & 0 & 6 & 0 & 0 & 0 & 0 & 0 & 0 & 0 & 4 & 5 \\
\hline Gefitinib/erlotinib $(n=2)$ & 0 & 2 & 0 & 0 & 0 & 0 & 0 & 0 & 0 & 1 & 2 \\
\hline
\end{tabular}

Abbreviations: EGFR, epidermal growth factor receptor; ICU, Intensive care unit; IP, interstitial pneumonitis; GI, Gastrointestinal; NSCLC, non-small cell lung cancer; TKI, tyrosine-kinase inhibitor; SCLC, small cell lung cancer

Decisions regarding the type and timing of anticancer therapy for the subject subpopulation are extremely complex, and only limited data exist regarding their impact $[15,16,22,23]$. Generally, lung cancer patients with poor PS are precluded from chemotherapy; BSC or palliative care is usually considered for these patients $[13,24]$. A few studies have suggested that immediate chemotherapy may be 
beneficial for selected critically ill cancer patients with newly diagnosed malignancies $[16,25,26]$. For example, chemotherapy might benefit selected newly diagnosed SCLC patients with cancer-related respiratory failure in the ICU, allowing relatively early extubation and ICU discharge [15].

In the present study, multivariate analysis revealed that lung cancer patients receiving chemotherapy might have longer ICU and in-hospital survivals than patients receiving BSC, regardless of histologic type or disease metastasis. Mechanical ventilation was also more common among patients in the treatment groups and was associated with longer ICU survival. In contrast, patients with DNR orders were more common in the BSC group than in the chemotherapy group and were associated with diminished ICU and hospital survival. Lung cancer patients receiving various first-line chemotherapies, in the ICU, also demonstrated better weaning rates than those receiving EGFR-TKIs and only BSC. The higher weaning rate associated with chemotherapy might be attributed to the highly chemoresponsive characteristics of treatment-naïve SCLC patients $(n=8)$ and some NSCLC patients $(n=2)$, similar to previously reported indications that combined full-code management and cancer chemotherapy might have short-term survival benefits for selected lung cancer patients [16,24-27]. Further study is warranted to evaluate the impact of chemotherapy and ICU support on long-term outcomes, quality of life, and cost effectiveness for this patient subgroup.

Patients with poor PS who receive chemotherapy also have higher risks of treatment-related toxicities [28]. We found that the most common grade 3 or 4 toxic effects noted in the chemotherapy group were neutropenia $(41.6 \%)$, thrombocytopenia $(20.8 \%)$, and gastrointestinal toxicity (20.8\%), similar to the general population of patients undergoing platinum-based chemotherapy for NSCLC [29] and SCLC [30]. The optimal chemotherapy regimen and dose for critically ill lung cancer patients has not been previously evaluated, and prospective clinical trials are needed to clarify the present findings.

Although there are studies reporting the poor prognosis of cancer patients requiring intensive care and mechanical ventilation support [8-12], based on the previous reports [15-16,25-26] and our findings, we suggest that treatment-naïve lung cancer patients could be considered as a distinct subgroup which might benefit from immediate chemotherapy, especially those with highly chemosensitive tumor (such as SCLC) presenting with cancer-related organ failures. Besides, NSCLC patients with wild-type EGFR or unknown mutation status, chemotherapy could be an effective treatment with relatively rapid response compared with targeted therapy [13].

Identifying the genetic mutation present in NSCLC patients is critical for targeted therapies [31,32], which are effective (improved progression-free survival, response rates, and quality of life relative to chemotherapy) in lung adenocarcinoma patients harboring the EGFR mutation [33,34]. Previous studies reported that first-line EGFR-TKIs provide greater clinical benefit to extremely poor PS NSCLC patients with short life expectancies than does BSC $[35,36]$. Others indicated that targeted molecular agents could be considered for use in cancer patients with extremely poor PS, even when they are receiving critical care [23]. In our study, critically ill lung cancer patients receiving EGFR-TKIs had better ICU and hospital survival than did BSC patients. Furthermore, significant differences in ICU and hospital survivals were not noted between the EGFR-TKI and chemotherapy groups. This observation might be attributed to the heterogeneity of lung cancer patients (SCLC and NSCLC patients) in the chemotherapy group and that most EGFR-TKI group NSCLC patients had wild type EGFR or an unknown molecular status. However, only one patient harboring positive EGFR mutation (Exon 19 Deletion) who required mechanical ventilation in EGFR-TKIs group was liberated from MV support and the majority of patients (16 of 17) in EGFR-TKI group harboring wild type EGFR mutation or an unknown molecular status who required MV support all were failure to wean from mechanical ventilation. These results were consistent with Hsia et al [37] that EGFR-TKIs for stage IV NSCLC patients requiring MV did not lead a better weaning outcome. In addition, three patients $(3 / 24,12.5 \%)$ in the EGFR-TKI group demonstrated gefitinib-related interstitial pneumonitis, consistent with previous reports that patients with poor PS might be more prone to drug toxicity than those with good PS, even in patients receiving targeted agents [38].

In this study, we were unable to determine the molecular mutation status of all patients due to their vulnerability to the invasive specimen collection procedures and possible some rare EGFR mutations could not be detected in the early period of targeted therapy era without sensitive detection methods (such as next-generation sequencing) [39-40]. Since this is a retrospective study that we could not clearly identify the decision criteria of using EGFR-TKIs for critically ill NSCLC patients without EGFR mutations. We supposed that clinicians might expect the benefits of EGFR-TKIs on the status of nonsmokers and non-squamous NSCLC, East Asian patients [41] with possible undetected less common EGFR mutations $[39,40]$. 
In our study, one patient with EGFR mutation-positive NSCLC received gefitinib had partial response and successfully weaned from MV and the other EGFR mutation- positive patient received best supportive care. In contrast, we found that up to $54.2 \%$ (13 of 24) patients in EGFR-TKI group with wild type EGFR mutation or unknown mutation status stopped using EGFR-TKIs due to disease progression and treated related interstitial pneumonitis. Only 2 patients with wild type EGFR mutation received EGFR-TKIs had partial response and one patient with an unknown mutation status had stable disease. Thus, the strategy of administering EGFR-TKIs to critically ill NSCLC patients should be carefully interpreted with only limited evidences [23,35-37]. Our findings suggest EGFR-TKIs therapy for NSCLC patients should be used according to the molecular status even under critical condition, which would be a better choice [13, 33-35]. Further research is warranted to evaluate the impact of various targeted therapies for critically ill NSCLC patients with known mutation status.

In real-world practice, the risks and benefits of administrating anticancer therapies for treatment-naïve, lung cancer patients requiring intensive care usually be evaluated by oncologists and intensivists to identify the patients who are most likely to have favorable outcomes. Besides, the further communication among medical team and the patients or the families is also very important, which especially focuses on informing the possible complications and discussing the satisfaction of their long-term quality of life [42]. Based on the previous studies $[15,16,22-27,33-38,42]$ and our findings, we suggest that treatment-naïve lung cancer can be treated with chemotherapy under critical condition for some selected patients, particularly those with SCLC or wild-type EGFR / unknown mutation status NSCLC and patients who required MV support but without DNR order. In addition, we also suggest that EGFR-TKIs could be only considered for the critically ill lung cancer patients with positive EGFR mutations.

The present study has several limitations. First, we analyzed a small number of highly selected, critically ill lung cancer patients with multiple complex confounding factors in a single-center, observational study. Thus, generalization of our findings will require further large-scale investigation. Second, data from patients with various types of lung cancer were pooled, possibly clouding the separate chemotherapeutic benefits for critically ill NSCLC and SCLC patients. Third, the benefit of anticancer therapies in ICU should be cautiously interpreted because of the retrospective nature of this study with selection bias and the possible treatment related adverse events may have been missed from the coding problems. Fourth, we could not evaluate patient clinical responses following treatment, due to their high mortality rates.

\section{Conclusions}

In conclusion, for newly diagnosed, critically ill lung cancer patients, chemotherapy and targeted therapy might provide short-term survival benefits and might be considered for selected patients. Further studies are needed to evaluate the benefits of combined ICU management and immediate anticancer therapy for treatment-naïve lung cancer patients.

\section{Acknowledgements}

The authors thank Hung-Chueh Peng and Kuei-Pin Chung for their assistance in data collection and helpful comments.

\section{Competing Interests}

None of the authors has a financial relationship with a commercial entity that has an interest in the subject of this manuscript.

\section{References}

[1] Jemal A, Siegel R, Ward E, et al. Cancer statistics, 2009. CA Cancer J Clin 2009; 59: 225-249.

[2] Boussat S, El'rini T, Dubiez A, et al. Predictive factors of death in primary lung cancer patients on admission to the intensive care unit. Intensive Care Med 2000; 26: 1811-1816.

[3] Lin YC, Tsai YH, Huang CC, et al. Outcome of lung cancer patients with acute respiratory failure requiring mechanical ventilation. Respir Med 2004; 98: 43-51.

[4] Reichner CA, Thompson JA, O'Brien S, et al. Outcome and code status of lung cancer patients admitted to the medical ICU. Chest 2006; 130: 719-723.

[5] Soares M, Darmon M, Salluh JI, et al. Prognosis of lung cancer patients with life-threatening complications. Chest 2007; 131: 840-846.

[6] Roques S, Parrot A, Lavole A, et al. Six-month prognosis of patients with lung cancer admitted to the intensive care unit. Intensive Care Med 2009; 35: 2044-2050.

[7] Soubani AO, Ruckdeschel JC. The outcome of medical intensive care for lung cancer patients: the case for optimism. J Thorac Oncol 2011;6:633-638.

[8] Adam AK, Soubani AO. Outcome and prognostic factors of lung cancer patients admitted to the medical intensive care unit. Eur Respir J 2008; 31: 47-53.

[9] Ewer MS, Ali MK, Atta MS, et al. Outcome of lung cancer patients requiring mechanical ventilation for pulmonary failure. JAMA 1986; 256: 3364-3366.

[10] Müller AM, Gazzana MB, Silva DR. Outcomes for patients with lung cancer admitted to intensive care units. Rev Bras Ter Intensiva 2013; 25: 12-16.

[11] Toffart AC, Minet C, Raynard B, et al. Use of intensive care in patients with nonresectable lung cancer. Chest 2011; 139: 101-108.

[12] Kim YJ, Kim MJ, Cho YJ, et al. Who should be admitted to the intensive care unit? The outcome of intensive care unit admission in stage IIIB-IV lung cancer patients. Med Oncol 2014; 31: 847.

[13] Ettinger DS, Akerley W, Borghaei $\mathrm{H}$, et al. Non-small cell lung cancer, version 2.2013. J Natl Compr Canc Netw 2013; 11: 645-653.

[14] Levy B, Saxena A, Schneider BJ. Systemic therapy for small cell lung cancer. J Natl Compr Canc Netw 2013; 11: 780-787.

[15] Jennens RR, Rosenthal MA, Mitchell P, et al. Outcome of patients admitted to the intensive care unit with newly diagnosed small cell lung cancer. Lung Cancer 2002; 38: 291-296.

[16] Darmon M, Thiery G, Ciroldi M, et al. Intensive care in patients with newly diagnosed malignancies and a need for cancer chemotherapy. Crit Care Med 2005; 33: 2488-2493.

[17] Centers for Disease Control and Prevention (CDC). Cigarette smoking among adults-United States, 2006. MMWR Morb Mortal Wkly Rep 2007; 56: 1157-1161.

[18] Knaus WA, Draper EA, Wagner DP, et al. APACHE II: a severity of disease classification system. Crit Care Med 1985; 13: 818-829. 
[19] Edge SB. AJCC cancer staging manual. New York, NY: Springer; 2010.

[20] Travis WD. Pathology and genetics of tumours of the lung, pleura, thymus and heart. Oxford, UK: Oxford University Press, 2004.

[21] CTC. Cancer Therapy Evaluation Program. 1; Revised March 23, 1998. Common Toxicity Criteria, Version 2.0. DCTD. 1999.

[22] Song JU, Suh GY, Chung MP, et al. Risk factors to predict outcome in critically ill cancer patients receiving chemotherapy in the intensive care unit. Support Care Cancer 2011; 19: 491-495.

[23] Ahn HK, Jeon K, Yoo H, et al. Successful treatment with crizotinib in mechanically ventilated patients with ALK positive non-small-cell lung cancer. J Thorac Oncol 2013; 8: 250-253.

[24] Paesmans M, Sculier JP, Libert P, et al. Prognostic factors for survival in advanced non-small-cell lung cancer: univariate and multivariate analyses including recursive partitioning and amalgamation algorithms in 1,052 patients. The European Lung Cancer Working Party. J Clin Oncol 1995; 13: 1221-1230.

[25] Benoit DD, Depuydt PO, Vandewoude KH, et al. Outcome in severely ill patients with hematological malignancies who received intravenous chemotherapy in the intensive care unit. Intensive Care Med 2006; 32: 93-99.

[26] Azoulay E, Afessa B. The intensive care support of patients with malignancy: do everything that can be done. Intensive Care Med 2006; 32: 3-5.

[27] Darmon M, Azoulay E. Critical care management of cancer patients: cause for optimism and need for objectivity. Curr Opin Oncol 2009; 21: 318-326.

[28] White SC, Lorigan P, Middleton MR, et al. Randomized phase II study of cyclophosphamide, doxorubicin, and vincristine compared with single-agent carboplatin in patients with poor prognosis small cell lung carcinoma. Cancer 2001; 92: 601-608.

[29] Socinski MA, Morris DE, Masters GA, et al. Chemotherapeutic management of stage IV non-small cell lung cancer. Chest 2003;123 (1 Suppl):226S-243S.

[30] Okamoto H, Watanabe K, Kunikane H, et al. Randomised phase III trial of carboplatin plus etoposide vs split doses of cisplatin plus etoposide in elderly or poor-risk patients with extensive disease small-cell lung cancer: JCOG 9702. Br J Cancer 2007; 97: 162-169.

[31] Travis WD, Brambilla E, Noguchi M, et al. International Association for the Study of Lung Cancer/American Thoracic Society/European Respiratory Society International Multi-Disciplinary Classification of Lung Adenocarcinoma. J Thorac Oncol 2011; 6: 244-285.

[32] Blackhall F, Thatcher N, Booton R, et al. The impact on the multi-disciplinary team of molecular profiling for personalized therapy in non-small cell lung cancer. Lung Cancer 2013; 79:101-103.

[33] Mok TS, Wu YL, Thongprasert S, et al. Gefitinib or carboplatin-paclitaxel in pulmonary adenocarcinoma. N Engl J Med 2009; 361: 947-957.

[34] Rosell R, Carcereny E, Gervais R, et al. Erlotinib versus standard chemotherapy as first-line treatment for European patients with advanced EGFR mutation-positive non-small-cell lung cancer (EURTAC): A multi-centre, open-label, randomised phase 3 trial. Lancet Oncol 2012; 13: 239-246.

[35] Inoue A, Kobayashi K, Usui K, et al. First-line gefitinib for patients with advanced non-small-cell lung cancer harboring epidermal growth factor receptor mutations without indication for chemotherapy. J Clin Oncol 2009; 27: 1394-1400.

[36] Lee YJ, Kim HT, Han JY, et al. First-line gefitinib treatment for patients with advanced non-small cell lung cancer with poor performance status. J Thorac Oncol 2010; 5: 361-368.

[37] Hsia TC, Tu CY, Chen HJ et al. The impact of rescue or maintenance therapy with EGFR TKIs for Stage IIIb-IV non-squamous non-small-cell lung cancer patients requiring mechanical ventilation. BMC Anesthesiol. 2014; 16: 14:55

[38] Hotta K, Inoue A, Kiura K, et al. Gefitinib should be cautiously administered to poor performance status patients with non-small-cell lung cancer: results from a prospective feasibility study. Lung Cancer 2005; 50: 413-415.

[39] Yoshihisa K and Tetsuya M. Not all epidermal growth factor receptor mutations in lung cancer are created equal: Perspectives for individualized treatment strategy. Cancer Sci 2016; 107: 1179-1186.

[40] Lin YT, Liu YN, Wu SG, Yang JC, Shih JY. Epidermal Growth Factor Receptor Tyrosine Kinase Inhibitor-sensitive Exon 19 Insertion and Exon 20 Insertion in Patients With Advanced Non-Small-cell Lung Cancer. Clin Lung Cancer. 2016 Dec 28. pii: S1525-7304(16)30390-4.

[41] Thatcher N, Chang A, Parikh P, et al. Gefitinib plus best supportive care in previously treated patients with refractory advanced non-small-cell lung cancer: results from a randomised, placebo-controlled, multicentre study (iressa survival evaluation in lung cancer). Lancet 2005, 366: 1527-1537.

[42] Tanvetyanon T. Consideration before administering cytotoxic chemotherapy to the critically ill. Crit Care 2005; 33: 2689-2691. 